Cite as: Stephane H Maes, (2020), "More Matter Than Antimatter, All Falling Down", viXra:2010.0121v2, https://vixra.org/pdf/2010.0121v2.pdf, https://shmaesphysics.wordpress.com/2020/07/05/more-matter-thanantimatter-all-falling-down/, July 5, 2020. (V2: April 8, 2021)

\title{
More Matter Than Antimatter, All Falling Down
}

\author{
Stephane H. Maes ${ }^{1}$
}

July 5,2020

(This is V.2-4/8/2021)

\begin{abstract}
:
In a multi-fold universe, gravity emerges from Entanglement through the multi-fold mechanisms. As a result, gravity-like effects appear in between entangled particles that they be real or virtual. Long range, massless gravity results from entanglement of massless virtual particles. Entanglement of massive virtual particles leads to massive gravity contributions at very smalls scales. Multi-folds mechanisms also result into a spacetime that is discrete, with a random walk fractal structure and non-commutative geometry that is Lorentz invariant and where spacetime nodes and particles can be modeled with microscopic black holes. All these recover General relativity at large scales and semi-classical model remain valid till smaller scale than usually expected. Gravity can therefore be added to the Standard Model. This can contribute to resolving several open issues with the Standard Model.

In particular with chirality flips of fermions induced by gravity, right-handed neutrinos (and left-handed antineutrinos) can appear in flight and now acquire mass when encountering Higgs bosons. Because perturbatively selfgravity effects may be stronger for anti-neutrinos, the chirality flips in flight will trap longer in flight right-handed anti neutrinos than left-handed neutrinos; creating a matter antimatter asymmetry. While very small this can explain the dominance of matter of antimatter, hence why we exist.
\end{abstract}

As we visit the properties of antimatter, we also predict that, in a multi-fold universe, anti-matter is attracted by gravity, not repelled; something that is still an open issue today in Physics.

\section{Introduction}

The new preprint [1] proposes contributions to several open problems in physics like the reconciliation of General Relativity (GR) with Quantum Physics, explaining the origin of gravity proposed as emerging from quantum (EPREinstein Podolsky Rosen) entanglement between particles, detailing contributions to dark matter and dark energy and explaining other Standard Model mysteries without requiring New Physics beyond the Standard Model other than the addition of gravity to the Standard Model Lagrangian. All this is achieved in a multi-fold universe that may well model our real universe, which remains to be validated.

With the proposed model of [1], spacetime and Physics are modeled from Planck scales to quantum and macroscopic scales and semi classical approaches appear valid till very small scales. In [1], it is argued that spacetime is discrete, with a random walk-based fractal structure, fractional and noncommutative at, and above, Planck scales (with a 2-D behavior and Lorentz invariance preserved by random walks till the early moments of the universe). Spacetime results from past random walks of particles. Spacetime locations and particles can be modeled as microscopic black holes (Schwarzschild for photons and spacetime coordinates, and metrics between Reisner Nordstrom [2] and Kerr Newman [3] for massive and possibly charged particles - the latter being possibly extremal). Although surprising, [1] recovers results consistent with other like [4], while also being able to justify the

\footnotetext{
${ }^{1}$ shmaes.physics@gmail.com
} 
initial assumptions of black holes from the gravity or entanglement model. The resulting gravity model recovers General Relativity at larger scale, as a 4-D process, with massless gravity, but also with massive gravity components at very small scale that make gravity significant these scales. Semi-classical models also work well till way smaller scales that usually expected.

In this paper, we remain at a high level of discussion of the analysis and references are generic for the subjects. It makes the points accessible to a wider audience and keeps the door open to further papers or discussions devoted to details of interest. Yet, it requires the reader to review [1], as we do not revisit here all the details of the multifold mechanism or reconstruction of spacetime. More targeted references for all the material discussed here are compiled in [1].

It is worth noting that this paper is not just about evangelizing results published in [1]. The analysis presented here is new.

\section{2. $\mathrm{SM}_{\mathrm{G}}$ : The Standard Model with Multi-Fold Gravity}

[1] proposes that in a multi-fold universe, the Lagrangian is complemented by terms associated to gravity and entanglement (in the form of the sum of the attractive effective potentials) [1].

$$
\mathscr{L}_{\text {total }}=\mathscr{L}_{S M}+\mathscr{L}_{\text {Gravity-with-MF }}+\mathscr{L}_{\text {EPR }}+\mathscr{L}_{\text {Others } \ldots}
$$

The effect of gravity can be seen through the attractive potential contributions of all the energy sources. It can also been seen as expressing the Standard Model Lagrangian in curved spacetime (semi-classical point of view), considered valid, in multi-fold universes, till small scales.

EPR entanglement is not believed to often play a significant role, except in dark matter use cases [5].

The last term represents all other "New Physics" terms and we will consider it to be null.

\section{Chirality and Helicity flips induced by Gravity}

$[1,6]$ propose how gravity is responsible for chirality flips for fermions; thereby also explaining the mass of neutrino and the fate of right-handed neutrinos and left-handed anti-neutrinos by adding these oscillations to inflight neutrinos oscillations.

\section{The Matter dominance over Anti-matter}

Among the open problems in physics, one of the biggest mysteries of all may be the challenges in explaining why there is a (local or global - it depends on the explanation) excess of matter: why didn't all the matter and antimatter created at the big bang annihilate so that no matter or anti matter subsist (other than may be in disjoint regions where, from the beginning, there would have been inhomogeneities of matter and antimatter ratios). This is known as the problem of matter-antimatter asymmetry [7].

Different explanation paths have been followed as reviewed in [7], including in particular: (Besides for example the mirror anti-universe, anti-matter and matter dominated segregated regions or electric dipole moment hypotheses)

- Baryogenesis, as initially proposed by Sakharov [8] with GUTs (Grand Unification Theories) or within the Standard Model with CP and Baryon number symmetry violations 
- $\quad$ Leptogenesis [9] with Lepton number violation (with new physics or within the Standard Model with righthanded neutrinos)

- Axiogenesis, e.g. [10] as a result of the Peccei-Quinn symmetry breaking invoked to explain the strong CP violation problem [11].

Unfortunately, none of the approaches described in the bullet list above are congruent with our findings in a multifold universe [1]:

- As explained in [1,12], gravity induced smearing of the chirality flips are expected to ensure that baryon and lepton number symmetries are no more anomalous and therefore to be more stringently respected. This would count out baryogenesis and leptogenesis.

- $\quad$ As explained in $[1,13]$, most GUTs have been challenged by our findings.

- As explained in $[1,14]$, it is possible to explain the strong CP violation problem, with gravity effects that lower the mass of the up quark and therefore eliminate the QCD term responsible for CP violation in QCD; without the need for Peccei-Quinn symmetry breaking and hence the axion. In addition, [1,5] shows how dark matter can also be explained without the need of the axion. No axion would imply no axiogenesis, even if we do not forbid the Peccei-Quinn symmetry breaking to also exist.

- Let us be clear. We make no prediction for or against axions. But because we do not need them, it does not seem suitable to try to explain the matter-anti mater asymmetry using them.

- Occam razor's principle however would suggest that this reduce the plausibility of their existence.

- $\quad$ The other explanations are either not falsifiable / validated (e.g. mirror universe and multi-verses of matter and anti-matter) or not confirmed so far by observations (e.g. no observed gamma radiation that could be associated to annihilation at the boundary between differently matter or anti-matter dominated regions) or experimentation (e.g. no validation of the existence of electric dipoles within elementary particles or that a slew of CP violations ([23]-section direct violation for tracking and references up to the latest) that are believed not to be sufficient to account for the degree of matter-antimatter asymmetry in our universe [23]).

- $\quad$ Neutrino CP violations as such are still awaiting some form of confirmation [20, 24]. [25] is currently providing the strongest hint, so far, that CP symmetry would violated by the weak interactions of leptons. This explanation would essentially agree with our conclusion below, except that we propose a different explanation, from the conventional model with $\delta_{C P}[26,27]$, involving gravity to explain it. It may be warranted because $\delta_{C P}$ is still overly constrained to explain the matter-antimatter asymmetry $[25,26]$. In addition, the anomaly smears, in the presence of gravity, as discussed in the first bullet above $([1,12])$, render lepton number violations less acceptable in the presence of gravity. Our approach below does not conflict with $[1,12]$.

Of course, there is still hope that all the CP violations tracked so far can help [15] provide an explanation for the matter-antimatter asymmetry; but as mentioned or arguments in [1,12] are problematic for the hopes pinned on especially leptogenesis, even with the New Physics it often implies $[9,15]$.

It would be great if, besides potentially knocking out (some of the) conventional explanations, the multi-fold universe mechanisms could provide a viable alternative that explains the matter dominance in our universe. It could also be just an additional contribution to some or all of the mechanisms above that would lead to string enough effect to explain the matter-antimatter asymmetry.

\section{The left-handed anti-neutrino trap}

In a multi-fold universe, and based on section 3, because of gravity, left-handed neutrinos flip in flight into righthanded neutrino, and back, and right-handed anti-neutrino flip into left-handed anti-neutrino, and back. They interact only in their flavor state (as left-handed neutrinos or right-handed anti neutrinos), except for Higgs mechanisms. The former being because Higgs is a spacetime property (field), which is also why Higgs interactions 
are exceptions to the tenancy models of the folds (see [16]) and it explains why it resulted into giving mass to neutrinos à la [1,6] (Note 10/16/20: See also [22] for additional considerations).

If we consider the perturbative interaction model of the Feynman diagrams, we know that antiparticles amount to particles "going back in time", at infinitesimal perturbative scales. [17] justifies an arrow of time, so we will assume that the analysis is well defined (and disagree with views that anti-particles would just have a reversed time arrow, preferring to say they go back in time for an unambiguous direction of time). From the point of view of the multifold mechanisms behind the emergence of gravity from entanglement [1], it means that moving anti-neutrinos will feel a slightly stronger self-gravity effect (where massive gravity from multi-fold mechanisms is not as weak as conventionally) than neutrinos.

The self-effect refers to the fact that in a multi-fold universe, a particle is not a point particle but can for example be modeled as a quantum sized blackhole which (semiclassical view) moves it a spacetime that it contributes to curve (think backreaction). Therefore, it "self-interact (in fact a key contributing factor to the non-renormalizability of gravity, at least in background dependent models). In multi-fold universes, this effect is realized by the impact of all the past folds (history of emitted virtual particle pairs) on the particle.

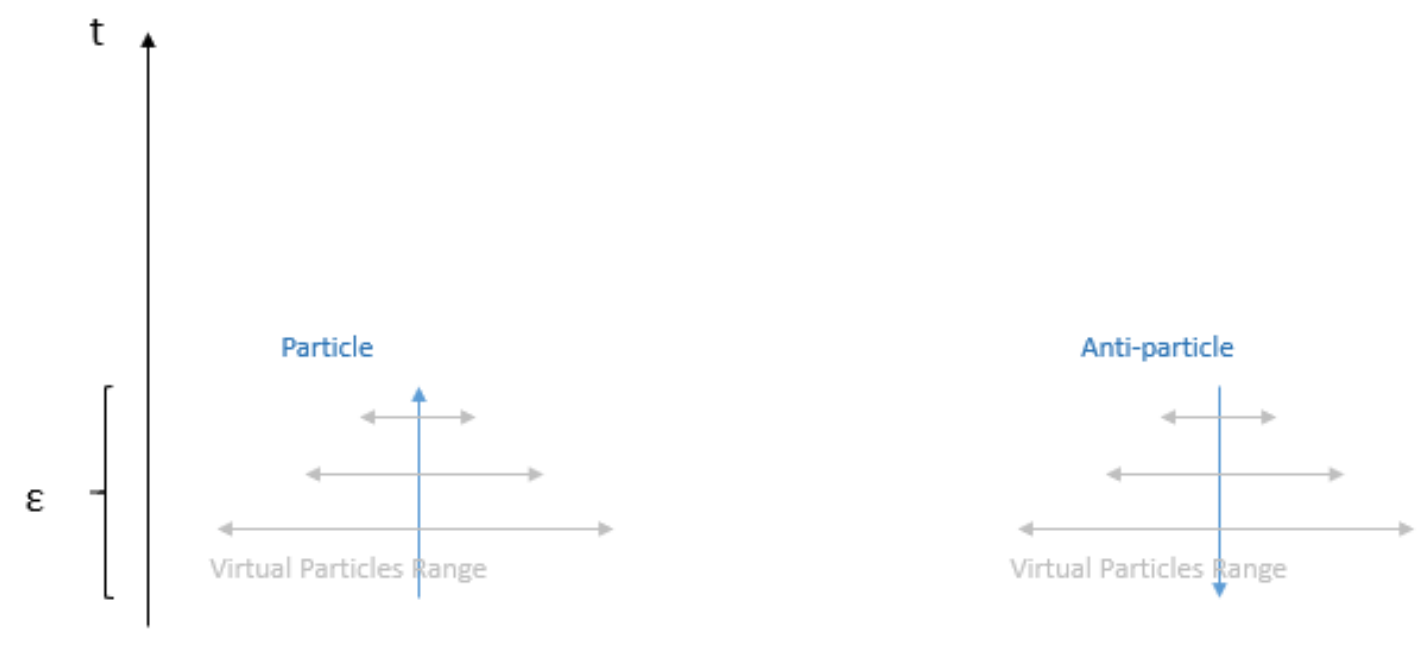

Figure 1: Moving particles in perturbative mode have at a given time a smaller cloud of entangled virtual pairs than anti-particles due to them going back in time (and remembering that neutrinos and anti-neutrinos move quasi at the speed of light. Note the arrow of time, that distinguish this reasoning of reversing the role of particles and antiparticles.

This is a new example of $\mathrm{C}$ and $\mathrm{CP}$ symmetry violations in neutrinos. The explanation is sketched on Figure 1 , where we need to remember that neutrinos move quasi at the speed of light and so it is analogous to a massless pattern as discussed in [1]. If time is infinitesimally reverted, the cloud of emitted virtual particles surrounding the anti-neutrino has a different shape and the particle encounters a larger range of multi-folds contributing to the self-attractive effective potential. As no particle is a point, but rather a (flattened because of its speed) uncertainty region, where we also find the microscopic black hole discussed in [1]. So there are effects of "self-gravity" felt and they could be different for anti-particles because they encounter local a different pattern of multi-fold folds associated emitted by the anti-particle. One can't just repat the opposite argument by exchanging particles and anti-particles because of the existence of an arrow of time [17] in multi-fold universe. All these effects are very, very small as $\varepsilon$ is infinitesimal. But it may matters for neutrinos. Anti-particles encounter in self-effects more multifolds than particles and so feel more strongly the effect. 
With this pattern, knowing that gravity flips chirality, we deduct that right-handed anti-neutrino have a slightly higher probability (because they feel a slightly stronger gravity effects) to flip into left-handed anti neutrinos than left-handed neutrinos to flip into right-handed neutrinos (and conversely exchanging the chiralities).

In flight, right-handed neutrinos or left-handed anti-neutrinos are not interacting, so they are not emitting the same number of virtual particles pairs that would increase the probability to flip back.

Iterating in flight: gravity flip, Higgs flips then again gravity flips produces less right-handed anti neutrinos than lefthanded neutrinos. This is the matter-antimatter that was expected. It is computed in Table 1 , where a>1 captures the stronger gravity flip for antimatter and $b<1$ captures the non-interaction of right neutrinos and left antineutrinos.

\begin{tabular}{|c|c|c|c|c|}
\hline L Neutrino & Gravity flip & R Neutrino & Higgs flip & L Anti Neutrino $x \mathrm{~b}$ \\
\hline R Neutrino & Gravity flip x b & L Neutrino $\times \mathrm{b}$ & Higgs flip & R Anti Neutrino $\times \mathrm{ab}$ \\
\hline L Anti Neutrino & Gravity flip x ab & R Anti Neutrino $x a b$ & Higgs flip & L Neutrino $x a b$ \\
\hline R Anti Neutrino & Gravity flip x a & L Anti Neutrino x a & Higgs flip & R Neutrino $x a$ \\
\hline
\end{tabular}

(A)

\begin{tabular}{|l|l|l|}
\hline L Anti Neutrino $x b$ & Gravity flip $x a b$ & R Anti Neutrino $x a^{2}$ \\
\hline R Anti Neutrino $x a b$ & Gravity flip $x a$ & L Anti Neutrino $x a^{2} b$ \\
\hline L Neutrino $x a b$ & Gravity flip & R Neutrino $x a b$ \\
\hline R Neutrino $x a$ & Gravity flip $x \mathrm{~b}$ & L Neutrino $x a b$ \\
\hline
\end{tabular}

(B)

Table 1: The flips in flights are row by row, with table (A) continued by table (B). The processes described (stronger effect for antimatter and less effect for non-interacting chirality) affects the probabilities of flips with $a>1$ and $b<1$. As a result, we have at the end: $a b$ ( $L$ Neutrinos) $>a b^{2}$ (R Anti Neutrinos); which completes our proof: more matter than antimatter. Table 1: The flips in flights are row by row, with table (A) continued by table (B). The processes described (stronger effect for antimatter and less effect for non-interacting chirality) affects the probabilities of flips with $a>1$ and $b<1$. As a result, we have at the end: $a b$ (L Neutrinos) $>a b^{2}$ ( $R$ Anti Neutrinos); which completes our proof: more matter than antimatter. (Note on 4/8/21: The table is corrected versus v.1 of the paper).

Neutrinos are so hard to observe and quantify that this variation in oscillations and availability for interactions has never been encountered experimentally. Yet, an indirect consequence should be that such an effect could account for a anti-neutrino deficit and hence a slight dominance of matter over antimatter (just as for example when hoping than longer conventional oscillations for anti-neutrinos (than for neutrinos) would make a similar impact in a tilted seesaw mechanism, as illustrated e.g. in [20]), and violate C and CP symmetries for neutrinos. It can contribute to explain the dominance of matter or be the main reason for it. To determine this latter aspect (be enough to account for the observed asymmetry), we would need a more quantitative model which is still work in progress.

Note that it would be worth seeing how this impacts also results like [24-26]. It is for future work.

Meanwhile, because of the approach, the lepton number Is not changed, in accordance with the arguments in [12] that this symmetry is to be more strictly respected when gravity is present; only availability of the lepton presents is skewed. 
The models in [1] are mostly qualitative so we cannot yet at this stage establish if effect is sufficient to be noticeable, and partially or fully account for the existence of matter over anti-matter. However, we should keep in mind that in the early age of the big bang, when the matter-antimatter asymmetry would result into a matter filled universe, energy and gravity were at their highest and flipping effects were more frequent across the board; therefore, probably for a while, exacerbating the effects on asymmetry. It may be strong enough or contribute enough and much more to other CP violations, to carry the day.

Note also that the proposed mechanism of a higher probability of flips applies to all fermions in $\mathrm{SM}_{\mathrm{G}}$ and reflects another example of $\mathrm{C}$ and $\mathrm{CP}$ symmetry violations by gravity with multi-fold mechanism beyond what had been considered in [1]. Differences in flips encountered decrease significantly as the fermions move at speed significantly lower than $c$, and more balanced longitudinal contributions of multi-fold (no more non-existent when not more at $c$ ) probably also renders the effect way smaller for more massive fermions other than neutrinos.

We have not evaluated yet if this has other effects due to, for example, the standard model chirality symmetry breaking. But the effects discussed here really result from the absence of interacting left-handed anti-neutrinos (and right-handed neutrinos); something that only affects neutrinos. So the effect analyzed here only applies to neutrinos. Tracking other effects, if any, are for future work.

The $\mathrm{C}$ and $\mathrm{CP}$ violations discussed here do not impact the gravity interaction with other particles or the conclusions on the equivalence principle [21]. It is also different from the symmetry breaking considerations for multi-fold mechanism and gravity discussed in [1].

Modeling and observing $\mathrm{C}$ and $\mathrm{CP}$ violations with neutrinos has been a long-sought objective of many projects. We again showed how adding non negligible gravity at small scale in a multi-fold universe can potentially help address open problems in Physics. Note that it is not clear, at the difference of other open problems, if just non-negligible gravity at small scales would work without mechanisms like the multi-fold mechanisms. Indeed, it is the way that the attractive effective potential is built that creates the $\mathrm{C}$ and $\mathrm{CP}$ symmetry violations.

Note 10/15/2020: Related impact of such proposal is also discussed in [22]. Such effects, if correct, could combine with what we propose in the present paper.

\section{Note on antimatter anti-gravity}

No difference should exist in attraction due to gravity for antimatter vs. matter. What we described above is only in terms of flipping effects due to self-gravity.

Because of the multi-fold mechanisms proposed in [1], the effects of the multi-folds is always in terms of attractive effective potential or positive curvature. It implies that, in a multi-fold universe, antimatter will also be attracted by gravity or entanglement that it be towards matter or antimatter. There simply is no other option with multifold mechanisms.

We expect that this will be confirmed by AEGIS and GBAR at LHC [18]. Today's early results of these experiments cannot yet answer definitively this question. This addresses another debate in Physics as reviewed in [19] and aligns with the other arguments in favor of always attractive behaviors, presented in [19].

If this prediction were to be invalidated, it would force us to reconsider aspects of the multi-fold mechanisms.

\section{Conclusions}


Our analysis is for a Multi-fold universe as in [1]. [1] details arguments and ways to check its relationship with the real universe. Besides properties that can be experimentally verified (in the future because of the macroscopic weakness of gravity and gravity like effects for entangled systems), [1] shows how the multi-fold mechanisms and behaviors are in many aspects in today's conventional physics, that, at times, anticipates the behaviors modeled of a multi-fold universe. In addition, [1] explains many results obtained in gravity, quantum mechanics, General Relativity, superstring theory, Loop Quantum Gravity and the AdS/CFT correspondence conjecture. All these works attempt to come up with models for the real universe. It is at least a good sign that [1] may provide an interesting model of the real universe.

Other theories showing that gravity is relevant at the level of the standard model, can repeat the chirality flip argument but may not be able to repeat the arguments for the increase in self-gravity effects for anti-neutrinos. They may also miss the argument of sections 6 , that ensures a priori only gravity attraction even for antimatter. Yet other arguments already exist to that effect anyway [19].

If our proposal were not validated by experience, it would not invalidate the multi-fold mechanisms and the proposal that gravity emerges from entanglement as detailed in [1]. The analysis, presented here, builds on [1], as a plausible consequence of it, but it is not a condition for validation of multi-fold universes.

We believe that [1] makes a compelling case for the consistency of its multi-fold proposal. The present paper shows how the mechanisms of multi-fold universes can help address the challenges with the dominance of matter over antimatter.

We explain that in a multi-fold universe, right-handed anti-neutrino have their chirality flipped by gravity while in flight and they get trapped slightly more often into the resulting in flight left-handed anti-neutrino mode that does not interact. As a result, and without New Physics, we can explain the dominance of matter over antimatter while preserving the lepton number symmetry and introduced a new $\mathrm{C}$ and $\mathrm{CP}$ violation for neutrinos and gravity. We also saw that multi-fold mechanisms ensure that antimatter, falls like matter (towards matter as well as antimatter).

This, along with similar results in $[1]$ and $[6,14]$, makes a strong case for more seriously considering the implications of adding gravity to the Standard Model to obtain $\mathrm{SM}_{\mathrm{G}}$, as a way to contribute to addressing open issues and offer better alternatives to New Physics speculations. This goes hand in hand with recognizing that this also implies the need to seriously consider that gravity may not always be negligible at the Standard Model scales as proposed in [1].

References: (most references come from popular science to make the discussion more approachable)

[1]: Stephane H. Maes, (2020) “Quantum Gravity Emergence from Entanglement in a Multi-Fold

Universe", viXra:2006.0088v1, (June 9, 2020).

[2]: https://en.wikipedia.org/wiki/Reissner\%E2\%80\%93Nordstr\%C3\%B6m metric

[3]: https://en.wikipedia.org/wiki/Kerr-Newman metric

[4]: Burinskii, Alexander, (2008), "The Dirac-Kerr-Newman electron", arXiv:0507109v4

[5]: Stephane H Maes, (2020), "Explaining Dark Matter Without New Physics?", viXra:2006.0261v1,

https://shmaesphysics.wordpress.com/2020/06/21/explaining-dark-matter-without-new-physics/, June 21, 2020.

[6]: Stephane H Maes, (2020), "Right-handed neutrinos? Mass? Ask

Gravity", viXra:2007.0018v1, https://shmaesphysics.wordpress.com/2020/06/21/right-handed-neutrinos-ask-

gravity/, June 21, 2020.

[7]: https://en.wikipedia.org/wiki/Baryon asymmetry

[8]: https://en.wikipedia.org/wiki/Baryogenesis

[9]: https://en.wikipedia.org/wiki/Leptogenesis

[10]: Raymond T. Co, Keisuke Harigaya, (2019), “Axiogenesis", arXiv:1910.02080v2. 
[11]: R. D. Peccei and Helen R. Quinn, (1977), "CP Conservation in the Presence of Pseudoparticles", Phys. Rev. Lett. 38, 1440

[12]: Stephane H Maes, (2020), "Gravity Induced Anomalies Smearing in Standard Model so that Protons May Never Decay, Except in Black Holes ", viXra:2006.0128v1, https://shmaesphysics.wordpress.com/2020/06/12/protons-may-never-decay-except-in-black-holes/ , June 13, 2020.

[13]: Stephane H Maes, (2020), "Ultimate Unification: Gravity-led Democracy vs. UberSymmetries", viXra:2006.0211v1, https://shmaesphysics.wordpress.com/2020/06/16/ultimate-unification-gravityled-democracy-vs-uber-symmetries/, June 16, 2020.

[14]: Stephane H Maes, (2020), "Strong CP Violation Tamed in The Presence of Gravity", viXra:2007.0025v1, https://shmaesphysics.wordpress.com/2020/06/23/strong-cp-violation-tamed-inthe-presence-of-gravity/, June 21, 2020.

[15]: https://en.wikipedia.org/wiki/CP violation

[16]: Stephane H Maes, (2020), "No Gravity Shield in Multi-folds Universes", viXra:2010.0032v1, https://shmaesphysics.wordpress.com/2020/06/26/no-gravity-shields-in-multi-foldsuniverses/, June 26, 2020.

[17]: Stephane H Maes, (2020), "Entanglement Concretizes Time in a Multi-fold Universe", viXra:2010.0083v1, https://shmaesphysics.wordpress.com/2020/06/28/entanglement-concretizes-time-in-a-multifold-universe/, June 28, 2020.

[18]: https://en.wikipedia.org/wiki/Antiproton Decelerator

[19]: https://en.wikipedia.org/wiki/Gravitational interaction of antimatter

[20]: https://www.quantamagazine.org/do-neutrinos-explain-matter-antimatter-asymmetry-20160728/

[21]: Stephane H Maes, (2020), "Derivation of the Equivalence Principle in a Multi-fold

Universe", viXra:2010.0090v1, https://shmaesphysics.wordpress.com/2020/06/29/derivation-of-the-equivalenceprinciple-in-a-multi-fold-universe/, June 19, 2020.

[22]: Stephane H Maes, (2020), "No Conventional Sterile Neutrinos In a Multi-fold Universe: just SMG business as usual", https://shmaesphysics.wordpress.com/2020/10/02/no-conventional-sterile-neutrinos-in-a-multi-folduniverse-just-smg-business-as-usual/, October 1, 2020.

[23]: Wikipedia, "CP violation", https://en.wikipedia.org/wiki/CP violation. Retrieved for this paper on October 16, 2020.

[24]: Johanna L. Miller, (2020), "Closing in on neutrino CP violation", Physics Today in Research \& Technology, in 23 Apr 2020, https://physicstoday.scitation.org/do/10.1063/pt.6.1.20200423a/full/. Retrieved for this paper on October 16, 2020.

[25]: K. Abe, et al., (2019), "Constraint on the Matter-Antimatter Symmetry-Violating Phase in Neutrino Oscillations", arXiv:1910.03887v2.

[26]: M.C. Gonzalez-Garcia, M. YokoyamaParticle, (2019), "14. Neutrino Masses, Mixing, and Oscillations", in "Review of Particle Physics", Particle Data Group, https://pdg.lbl.gov/2019/reviews/rpp2018-rev-neutrino$\underline{\text { mixing.pdf }}$

[27]: Harald Fritzsch, Zhi-zhong Xing, (2001), "How to Describe Neutrino Mixing and CP Violation", arXiv:hepph/0103242v2 